\title{
¿D e qué mueren los chilenos hoy?: perspectivas para el largo plazo
}

\author{
Giorgio Solimano $C^{1}$, Marinella Mazzei P2a. \\ Which are the causes \\ of death among Chileans today? \\ Long-term perspectives
}

During the last decades, Chile experienced substantial socioeconomic, epidemiological and demographic changes. These resulted, among other consequences, in a deceleration of population growth, a notorious decrease in fertility rates, and one of the most rapid and deepest drop in general and infant mortality rates in the Latin American region. These changes resulted in a sustained increase of life expectancy and a substantial ageing of the Chilean population. This process is also changing the disease burden of the population. Infectious and perinatal diseases lost relevance as major causes of mortality, and have been replaced by chronic non transmissible diseases, specifically cardiovascular conditions and cancer, that are becoming the main causes of death. High blood pressure, cardiovascular risk, hypercholesterolemia, diabetes, overweight and obesity, smoking, sedentary lifestyle and depression will have a great impact on health conditions during the XXI century. These factors and a persistent social inequity will hinder the efforts to reduce the impact and consequences of chronic non transmissible, diseases in the Chilean population (Rev Méd Chile 2007; 135: 932-8).

(Key words: Cause of death; Chronic disease; Life expectancy; Public health)

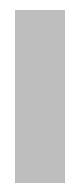
\footnotetext{
Universidad de Chile.

aSocióloga y Demógrafa
}

Recibido el 18 de enero, 2007. Aceptado el 13 de abril, 2007.

${ }^{1}$ Escuela de Salud Pública, Facultad de Medicina, Universidad de Chile. Santiago de Chile. ${ }^{2}$ División de Bioestadística y Demografía, Escuela de Salud Pública, Facultad de Medicina,

L as drásticas transformaciones que ha experimentado la región latinoamericana y Chile en particular, han sido consecuencia de los procesos de cambios socioculturales y económicos que han tenido lugar en las últimas décadas.

Desde inicios de la década de 1960, Chile ha visto modificar significativamente su estructura

Correspondencia a: Dr. Giorgio Solimano C. Escuela de Salud Pública, Facultad de Medicina, Universidad de Chile. Casilla 70012, Correo 7, Santiago. Fono: (56-2) 9786149. Fono/Fax (56-2) 7355582. E mail: gsolimano@med.uchile.cl etárea así como la transición de las causas de morbilidad y mortalidad. Son estos cambios los que están definiendo las tendencias demográficas y de salud para el siglo XXI.

El mejoramiento progresivo de las condiciones generales de vida de la población en el contexto de un acelerado proceso de urbanización, importante ampliación en la cobertura y calidad del saneamiento básico, mejor nutrición, mayor escolaridad, avances en el área médica e institucionalización de los sistemas nacionales de salud, constituyen el entorno que ha dado inicio a los 
notorios descensos en la mortalidad general $(11,9$ a 5,3 por mil habitantes entre 1960 y el año 2003), y especialmente de la mortalidad infantil $(106,4$ a $7,8$ por mil nacidos vivos en igual período $)^{1}$ (Figura 1).

Ello se ha reflejado, entre otros, en el incremento sostenido en el promedio de años de vida de nuestra población y en una transición sin precedentes del patrón de morbimortalidad ${ }^{2,3}$.

Así también, la población chilena ha visto incrementar su esperanza de vida al nacer desde un promedio de 57 años de edad a comienzos de la década de 1960, a unos 76 años en la actualidad, alcanzando incluso en la población femenina los 79 años en promedio de vida ${ }^{1}$. Las proyecciones demográficas que realizan tanto el Instituto Nacional de Estadística (INE) como el Centro Latinoamericano de Demografía (CELADE), estiman que para el quinquenio 2045-2050 la esperanza de vida al nacer aumentará hasta los 82 años de edad en promedio para ambos sexos, 79 años para los hombres y 85 años para las mujeres ${ }^{4,5}$.

En contraste con aquello, el país ha asistido a una disminución histórica en los niveles de fecundidad de la población, desde un promedio de 5,5 hijos por mujer en el período 1955-1960, a sólo 2 hijos en promedio en el período 2000-2005. Es de esperar que este promedio se estabilice en el orden de 1,9 hijos por mujer hacia el año 2015, es decir, un nivel algo inferior que aquel requerido para asegurar el reemplazo de la población a lo largo del tiempo (2,05 hijos en promedio por mujer) $)^{5-7}$

Así, a pesar de que el ritmo de crecimiento de la población se ha desacelerado, se espera que ésta llegue a un poco más de 20 millones de habitantes hacia mediados del presente siglo. Ello implicaría un aumento del orden de $31,2 \%$ en los próximos 50 años, muy distante del incremento de 153,2\% que experimentó el tamaño de la población nacional entre los años 1950 y 20004,5. Esta realidad demográfica ubica al Chile de hoy como un país en una etapa de transición epidemiológica y demográfica avanzada y en el segundo país de la región con mayor esperanza de vida al nacer y menor nivel de mortalidad infantil ${ }^{3-5,8-11}$.

Sin duda, que uno de los impactos más relevantes de este contexto de recomposición sociodemográfica es el cambio en la estructura por edad de la población y con ello el gradual

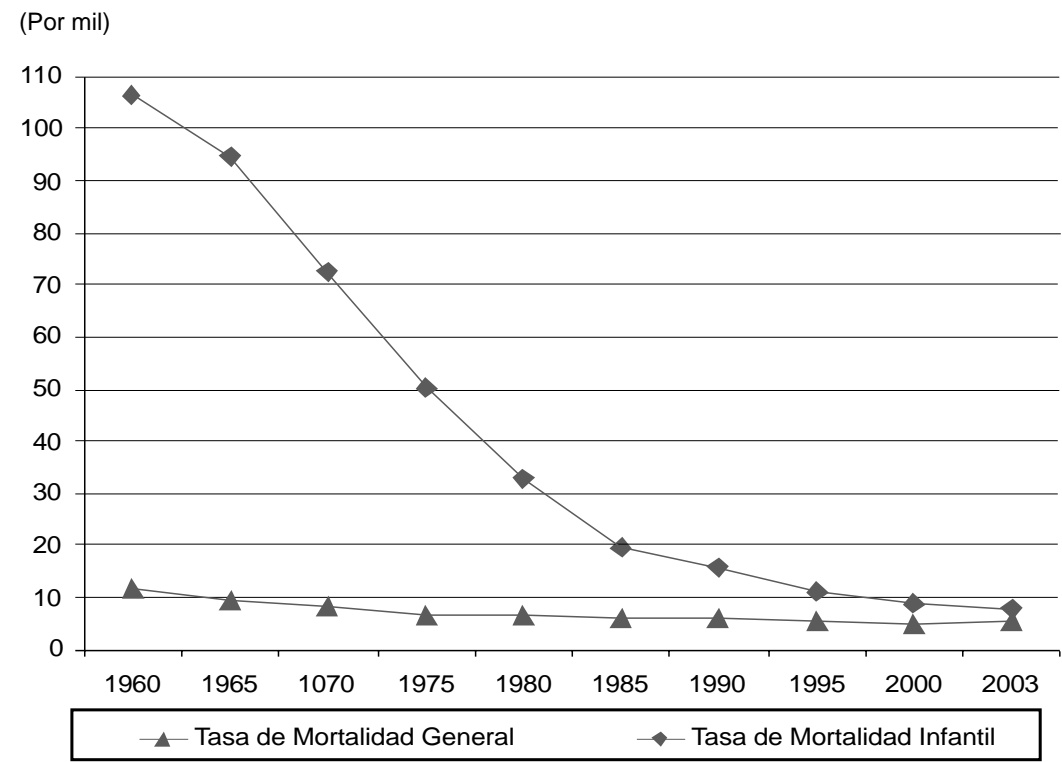

Fuente: CEPAL/CELADE: Boletín demográfico Nº73. América Latina: Proyecciones de población 1970-2050. ONU, 2004

Figura 1. Chile: Tasa de mortalidad general e infantil 1960-2003. 
proceso de envejecimiento al que estamos asistiendo $2,4,7-9,11-13$.

Según el último Censo de Población y Vivienda realizado en Chile en el año 20024,7, la población mayor de 60 años representaría 11,4\% de la población total nacional, es decir, 400.000 más de los registrados en el Censo anterior del año 1992. Si esto lo relacionamos con el hecho de que la población menor de 5 años decreció en dicho período 3,3\%, no cabe duda que se está asistiendo en Chile a un cambio de la estructura por edad de la población nunca antes visto. Ello se expresaría en un decrecimiento sostenido de la población menor de 15 años, un incremento importante de aquélla entre los 15 y 59 años de edad y a un aumento también sostenido de aquélla mayor de 60 años; se espera que esta población represente en el año 2050 21,6\% de la población total. Esta última situación implicaría una relación aproximada de apenas 6 niños por cada 10 adultos mayores ${ }^{4,5,7-9}$.

La atención de las necesidades y demandas propias de esta población adulta mayor en todos los ámbitos sociales, públicos y privados, se hará sentir principalmente, según estimaciones del CELADE, en el primer cuarto del presente siglo
(2000-2025), al presentar una tasa de crecimiento de $3,3 \%$, mucho mayor a la que se espera se registre en el período siguiente (2025-2050), la que se ubicaría en $1,5 \% 5,13$. Ello constituiría una realidad tremendamente importante, puesto que hacer frente al tema del envejecimiento poblacional y su impacto es un desafío no de futuro, sino uno al cual debemos como sociedad hacernos cargo en este momento (Figura 2).

Adicionalmente, habría que destacar que dentro del grupo que conforma la población de adultos mayores, es el de edad más avanzada (75 y más años), aquel que presenta crecimiento más acelerado $4,5,13$, lo que contribuirá al desarrollo de un fenómeno de longevidad poblacional sin precedentes, que tendrá impacto en el ámbito social y particularmente en el de la salud con importantes implicancias económicas, tanto en el sector público como el privado. En este sentido, la Encuesta de Caracterización Socioeconómica (CASEN) del año 2003, nos expone como ha ido incrementando en forma sostenida desde el año 1996 la población que pertenece al sistema de seguro público de salud, junto al decrecimiento de aquella adscrita al sistema privado de ISAPRES $^{14}$ (Figura 3), desigual distribución que se

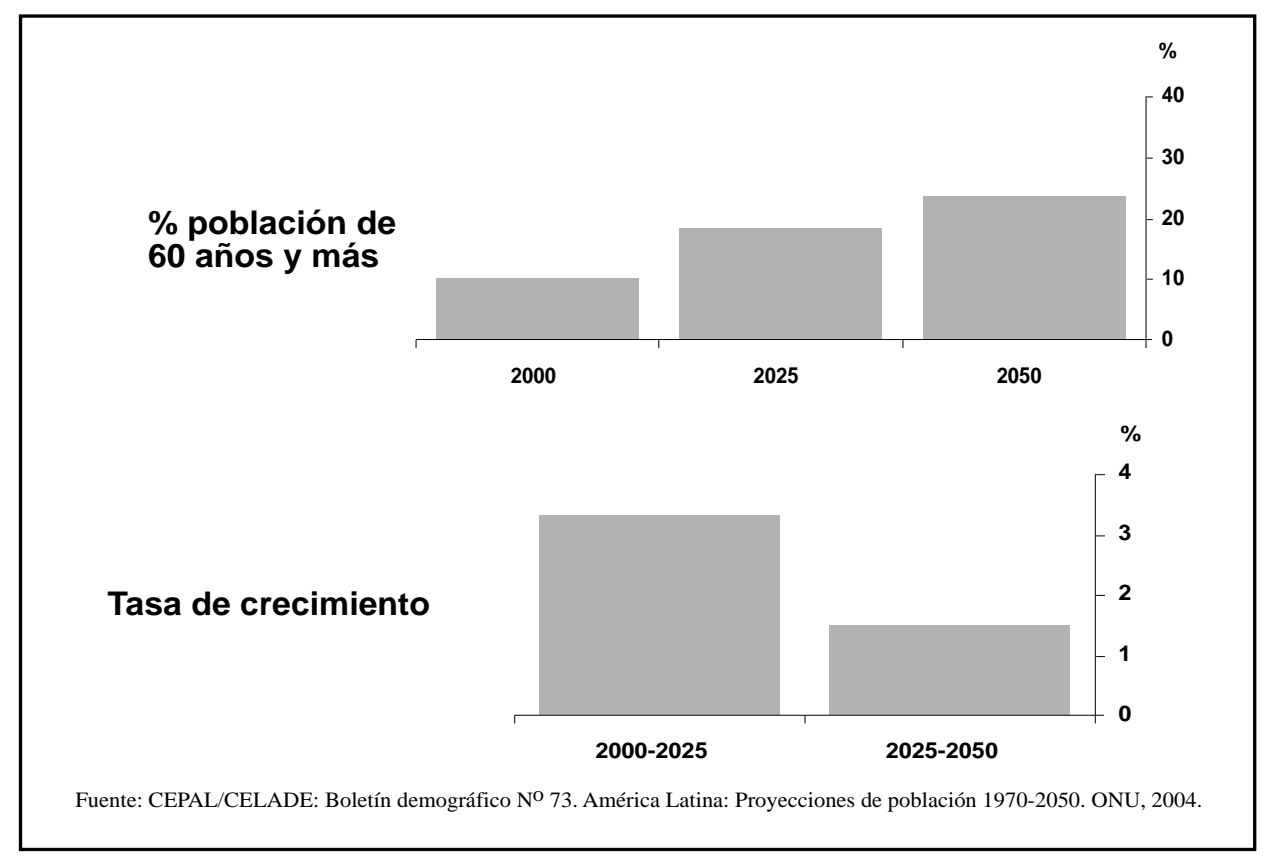

Figura 2. Chile: evolución de la población de 60 años y más, 2000-2050. 


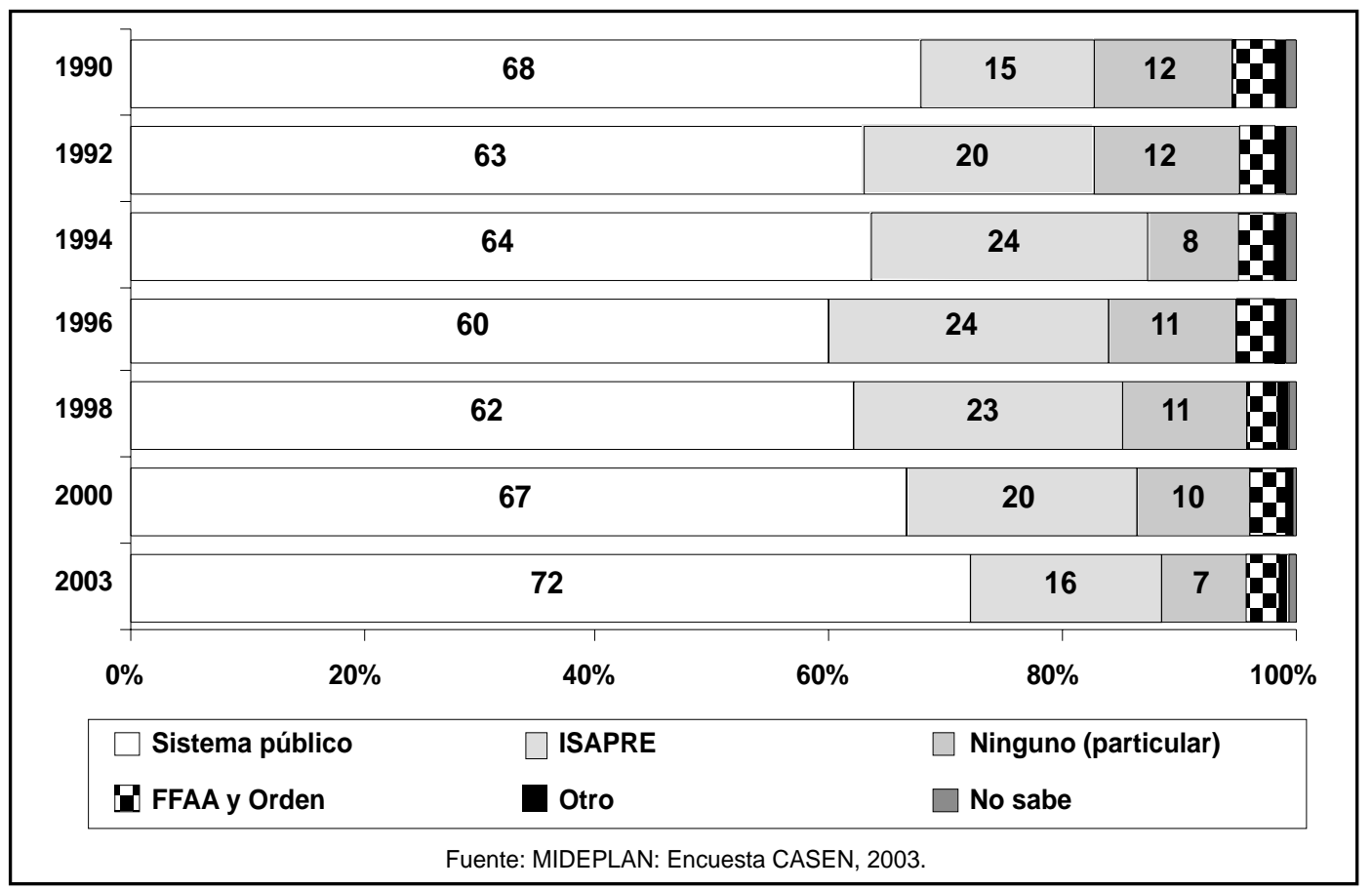

Figura 3. Chile: evolución de los beneficiaros por sistema previsional, 1990-2003.

profundiza en el segmento de población adulta mayor.

En el contexto sociodemográfico descrito, otra de sus principales consecuencias es el proceso de recomposición de la estructura de morbimortalidad: en 1969 el 44\% de la mortalidad correspondía a enfermedades infecciosas y perinatales, mientras que en el año 2001, 68\% correspondían a las enfermedades crónicas no transmisibles ${ }^{1,9,10}$.

De acuerdo a lo anterior: ¿de qué mueren los chilenos hoy?, ¿de qué se enferman?, ¿se enferman y mueren de lo mismo? Si bien en los últimos 50 años hemos asistido a un descenso significativo de las tasas de mortalidad, así también ha sufrido transformaciones el tipo de patologías que afectan a la población.

Desde mediados de la década de 1980, las enfermedades del sistema circulatorio y los tumores malignos constituyen las principales causas de muerte en el país, las que se presentan mayormente entre la población adulta y adulta mayor. Durante el año 2003, según estadísticas oficiales del Ministerio de Salud ${ }^{1,9}$, de las 83.672 defunciones registradas, más de la mitad de estas defunciones se debieron a este tipo de patologías $(28,4 \%$ y $23,5 \%$, respectivamente). En el mismo año le siguen en importancia aquellas denominadas causas externas de mortalidad y morbilidad, así como las enfermedades del sistema respiratorio (representando cada una 8,9\% del total de defunciones). La quinta causa de muerte está relacionada con trastornos del sistema digestivo (7,9\%).

Al observar el comportamiento de estas tasas en el período 1999-2003, se aprecia una disminución importante en la incidencia de las enfermedades del sistema respiratorio, al pasar de $14 \%$ en el año 1999 a 8,9\% en 2003. Por el contrario, se observa en igual período un incremento en las defunciones por tumores malignos (de 21,8\% a $23,5 \%)$, así como de las muertes relacionadas con el sistema digestivo (de 6,7\% a 7,9\%) ${ }^{9}$.

En cuanto al tipo de tumores, la principal incidencia la presentan aquellos del estómago, bronquios y pulmón y en tercer lugar los que 
afectan a la próstata. En la mujer, el cáncer mamario ocuparía el segundo lugar después del tumor del estómago y en tercer lugar el de la vesícula biliar ${ }^{9}$. Así también habría que destacar el importante diferencial entre sexos que presentan las tasas de mortalidad causadas por traumatismos, envenenamientos y algunas otras causas externas, siendo considerablemente superiores entre los hombres ${ }^{1,9}$.

Es de esperar que el nuevo perfil etáreo de la población, con una predominancia de población adulta y adulta mayor, favorezca un incremento tanto de patologías asociadas a estos grupos como de la elevación en los niveles de mortalidad.

Por su parte, al comparar el panorama de morbimortalidad del Chile de hoy con algunos de los principales resultados de la primera Encuesta Nacional de Salud realizada en el año $2003^{15}$ : 42\% de la población chilena mayor de 15 años fuma; casi 34\% tiene hipertensión arterial; 55\% presenta riesgo cardiovascular definido como alto y muy alto; $40 \%$ tiene su fracción protectora del colesterol disminuida; casi $23 \%$ padece síndrome metabólico; $4,2 \%$ es diabética; un alto porcentaje presenta sobrepeso u obesidad, y más de $90 \%$ de la población es sedentaria. Por su parte, los problemas de salud mental, en especial la depresión, también tienen una incidencia significativa especialmente entre la población femenina. Todo ello configura el ambiente de alto riesgo en que vive nuestra población, lo que hace aún más apremiante la intervención sobre estos y otros factores de riesgo asociados a este cambio de conductas y hábitos de consumo en el marco de nuevos estilos de vida.

Recientemente, el Ministerio de Salud ha realizado una evaluación del estado de avance a mitad de período de los Objetivos Sanitarios para la Década 2000-2010»6, cuya definición constituyó uno de los primeros pasos en el proceso de Reforma de Salud impulsado por el Presidente Ricardo Lagos durante su sexenio. Así, los «Objetivos Sanitarios»se agrupan en cuatro: I) Mejorar los logros sanitarios alcanzados; II) Enfrentar los desafíos derivados del envejecimiento de la población y de los cambios de la sociedad; III) Disminuir las desigualdades en salud; y IV) Proveer servicios acordes a las expectativas de la población ${ }^{17}$.

Esta evaluación es concordante con las estadísticas que hemos presentado en este artículo.
Observamos que se están cumpliendo satisfactoriamente tanto aquellas metas establecidas para las afecciones del niño y la mujer como para el de las enfermedades infecciosas, lo que no debe sorprendernos teniendo en cuenta los resultados exitosos de los programas materno infantiles y de control de las enfermedades infecto contagiosas en nuestro país desde hace décadas.

Sin embargo, la morbilidad y la mortalidad por enfermedades crónicas no transmisibles ha experimentado leves descensos o estancamiento durante los primeros 5 años de esta década ${ }^{16}$, en estrecha asociación con los factores de riesgo examinados y la permanencia de determinantes sociales de carácter estructural que no se modifican e incluso tienden a agravarse. Las persistentes diferencias en los niveles de escolaridad y de ingreso económico hacen que las brechas en los indicadores, aun cuando mejoran en término de tasas, se amplíen entre los extremos ${ }^{12,14,17,18}$ (Figura 4).

El escenario expuesto plantea importantes desafíos en dos ámbitos prioritarios. El primero guarda relación con la formulación de políticas, estrategias y programas de salud orientados a la promoción y protección de la misma, enfatizando el «cuidado de la salud»y no sólo la «atención a la enfermedad». Es precisamente en esta dirección que nuestro país tiene que avanzar en forma decidida. En otras palabras, se trata de cuidar y proteger la salud de la población, evitando o reduciendo los riesgos junto con el mejor tratamiento disponible de las distintas patologías, incluyendo las emergentes. El renovado énfasis en «e-conocer» los determinantes sociales en su dimensión individual y poblacional, dando especial importancia a la pobreza y a las grandes desigualdades existentes en Chile, se erige como un desafío urgente e ineludible.

Un segundo ámbito a considerar, es la necesidad imperiosa de formar y capacitar una fuerza de trabajo en salud que posea los conocimientos, competencias y habilidades para enfrentar los desafíos actuales y futuros aquí expuestos. En los últimos 20 años hemos asistido y en cierta medida seguimos asistiendo, a importantes reformas a la salud que han puesto el énfasis en el financiamiento y la gestión, olvidando que son los equipos de salud, conformados por equipos multiprofesionales, quienes tienen en sus manos su cuidado y protección. Contar con recursos huma- 


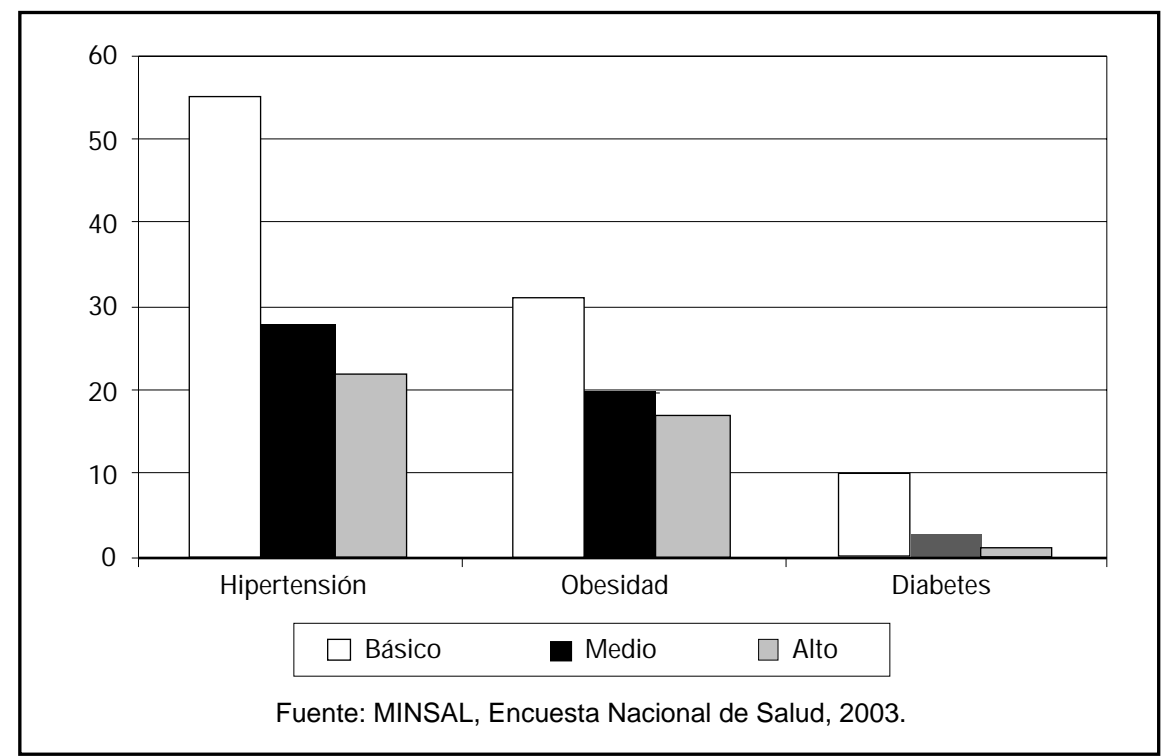

Figura 4. Chile: mortalidad por enfermedades crónicas según nivel educacional, 2003.

nos de alta calidad, en cantidad suficiente y que satisfagan las necesidades actuales y futuras, es de vital importancia.

Desafortunadamente en nuestro país carecemos de una política de recursos humanos en salud que permita establecer de manera informada $y$, utilizando metodologías modernas, las necesidades actuales y futuras de profesionales, el perfil requerido y muy especialmente los cambios que debe experimentar éste a medida que se producen modificaciones en las patologías prevalentes.

\section{REFERENCIAS}

1. INE. Anuario de Estadísticas Vitales. Instituto Nacional de Estadística, Santiago, 2003.

2. CEPAL/CELADE/UNFPA. Temas de Población y Desarrollo: transición demográfica, $\mathrm{N}^{\circ} 1$, Comisión Económica para América Latina, Centro Latinoamericano de Demografía y División de Población de Naciones Unidas, Santiago, 2005.

3. INE. Enfoques Estadísticos: mortalidad, №12, Instituto Nacional de Estadística, Santiago, 2001.

4. INE. Chile Hacia el 2050: proyecciones de población. Instituto Nacional de Estadística, Santiago, 2005.
Esta situación no es exclusiva de nuestro país, nos atrevemos a decir que es generalizada en el mundo, pero que existen posibilidades ciertas de abordarla con éxito si tomamos conciencia de su importancia en un país como el nuestro que cuenta con la capacidad, los recursos intelectuales, tecnológicos y materiales para implementar los programas de formación y capacitación que se requieren. El lograrlo constituye una tarea de todos, de la cual debemos hacernos cargo en forma decidida y responsable.

5. CELADE/CEPAL. Boletín Demográfico: América Latina y El Caribe, estimaciones y proyecciones de población 1950-2050, №73. Centro Latinoamericano de Demografía y Comisión Económica para América Latina, Naciones Unidas, Santiago, 2004.

6. CEPAL/CELADE/UNFPA. Temas de Población y Desarrollo: comportamiento reproductivo, №2, Comisión Económica para América Latina, Centro Latinoamericano de Demografía y División de Población de Naciones Unidas, Santiago, 2005. Instituto Nacional de Estadística, Santiago, 2005.

7. INE. Censo 2002. Síntesis de Resultados. Instituto Nacional de Estadística, Santiago, 2003. 
8. MazzeI M. Envejecimiento Poblacional en Chile y América Latina. Rev Chil Salud Pública 2003; 7: 104-5.

9. INE. Enfoques Estadísticos: información demográfica, $\mathrm{N}^{\circ} 28$, Instituto Nacional de Estadística, Santiago, 2005.

10. Szot J. La Transición Demográfico-Epidemiológica en Chile, 1960-2001. Rev Esp Salud Pública 2003; 77: 605-13.

11. CEPAL/CELADE/UNFPA. Temas de Población y Desarrollo: mortalidad, №4, Comisión Económica para América Latina, Centro Latinoamericano de Demografía y División de Población de Naciones Unidas, Santiago, 2005.

12. CEPAL/CELADE/BID. Impacto de las Tendencias Demográficas sobre los Sectores Sociales en América Latina: contribución al diseño de políticas y programas. Comisión Económica para América Latina, Centro Latinoamericano de DemografíaONU y Banco Interamericano de Desarrollo, Santiago, 1996.

13. GuZmán J. Envejecimiento y Desarrollo en América Latina y El Caribe. Serie Población y Desarro- llo. Centro Latinoamericano de Demografía y Comisión Económica para América Latina, Naciones Unidas, Santiago, 2002.

14. MIDEPLAN. Encuesta de Caracterización Socioeconómica de Chile (CASEN): 2003: resultados generales. Ministerio de Planificación, Santiago, 2004.

15. MINSAL. I Encuesta Nacional de Salud 2003. Ministerio de Salud de Chile, Santiago, 2004.

16. MINSAL Objetivos Sanitarios para la Década 2000-2010. Evaluación a mitad del Período: estado de avance en los objetivos de impacto. Ministerio de Salud de Chile, Santiago, 2006.

17. MINSAL Objetivos Sanitarios para la Década 2000-2010. Ministerio de Salud de Chile, Santiago, 2002.

18. Rodríguez J. Pobreza y Población: enfoques, conceptos y vínculos con las políticas públicas con especial referencia a la experiencia y situación en América Latina. Centro Latinoamericano de Demografía y Fondo de Población de Naciones Unidas, Santiago, 2006. 